\title{
The Influence of Distance on Radiant Exposure and Degree of Conversion Using Different Light- Emitting-Diode Curing Units
}

\author{
AO Al-Zain • GJ Eckert• JA Platt
}

\section{Clinical Relevance}

It may be necessary to double or triple exposure times when polymerizing at increasing clinically relevant distances from the base of the preparation.

\section{SUMMARY}

Objectives: To investigate the influence of curing distance on the degree of conversion (DC) of a resin-based composite (RBC) when similar radiant exposure was achieved using six different light-curing units (LCUs) and to explore the correlation among irradiance, radiant exposure, and DC.

Methods and Materials: A managing accurate resin curing-resin calibrator system was used to collect irradiance data for both top and bottom specimen surfaces with a curing distance of $2 \mathrm{~mm}$ and $8 \mathrm{~mm}$ while targeting a consistent top surface radiant exposure. Square nanohybrid-dual-photoinitiator $\mathbf{R B C}$

*Afnan O Al-Zain, BDS, MSD, PhD, King Abdulaziz University, Faculty of Dentistry, Restorative Dentistry Department, Jeddah, Saudi Arabia

George J Eckert, MS, Indiana University School of Medicine, Division of Biostatistics, Indianapolis, IN, USA

Jeffrey A Platt, DDS, MS, Indiana University, Department of Biomedical and Applied Sciences, Indianapolis, IN, USA

*Corresponding author: PO Box 80209, Jeddah 21589, Saudi Arabia; e-mail: alzain@kau.edu.sa

DOI: $10.2341 / 18-004-\mathrm{L}$ specimens $(5 \times 5 \times 2 \mathrm{~mm})$ were cured at each distance $(n=6 / \mathrm{LCU} /$ distance $)$. Irradiance and DC (micro-Raman spectroscopy) were determined for the top and bottom surfaces. The effect of distance and LCU on irradiance, radiant exposure, and $\mathrm{DC}$ as well as their linear associations were analyzed using analysis of variance and Pearson correlation coefficients, respectively $(\alpha=0.05)$.

Results: While maintaining a similar radiant exposure, each LCU exhibited distinctive patterns in decreased irradiance and increased curing time. No significant differences in DC values (63.21\%-70.28\%) were observed between the 2- and 8-mm distances, except for a multiple-emission peak LCU. Significant differences in DC were detected among the LCUs. As expected, irradiance and radiant exposure were significantly lower on the bottom surfaces. However, a strong correlation between irradiance and radiant exposure did not necessarily result in a strong correlation with DC.

Conclusions: The RBC exhibited DC values $>63 \%$ when the top surface radiant exposure was maintained, although the same values

\footnotetext{
"Used by permission. (C) Operative Dentistry, Inc. Transmission or reproduction of protected items beyond that allowed by fair use requires the written permission of Operative Dentistry, Inc."
} 
were not reached for all lights. A moderatestrong correlation existed among irradiance, radiant exposure, and DC.

\section{INTRODUCTION}

The polymerization effectiveness of light-cured resin-based composite (RBC) depends on its composition and the light's spectral characteristics. ${ }^{1,2}$ An RBC should receive the necessary power at the appropriate wavelengths for a sufficient amount of time to effectively activate polymerization. ${ }^{1-5}$ The amount of energy needed varies according to the RBC's composition, shade, and translucency. ${ }^{1-5}$

Manufacturers do not typically disclose all of their product components and concentrations. The manufacturer-recommended RBC curing time is typically based on testing the material in ideal laboratory conditions, in which a light-curing unit (LCU) guide tip is positioned as close as possible to the specimen, commonly at a distance of $0 \mathrm{~mm} .{ }^{5}$ However, in a clinical setting, a 0 -mm distance between the light guide and the restoration surface or cavity floor is not often attained. For example, the distance between the light guide tip and the cervical floor of a class II proximal box can reach up to $8 \mathrm{~mm}^{3}$ Increasing distance decreases the amount of irradiance (irradiance $\left[\mathrm{mW} / \mathrm{cm}^{2}\right]=$ power/surface area) ${ }^{1-6}$ and radiant exposure (radiant exposure $\left[\mathrm{J} / \mathrm{cm}^{2}\right]=$ irradiance $\times$ time $)^{1,2,5,6}$ received by an RBC. Consequently, using the same curing time as instructed by the manufacturer when the distance is increased can result in a lower radiant exposure than required for sufficient photoinitiator activation. ${ }^{3,7,8}$ This can lead to formation of a polymer network with less than ideal properties. ${ }^{2-4,9,10}$ In addition to decreased properties, insufficient polymerization can result in leaching of unreacted monomers into the oral environment and compromised restoration longevity. ${ }^{3,11-13}$ Therefore, increasing curing time may be needed as the curing distance increases from the RBC.

When maintaining radiant exposure, some RBCs exhibit similar properties, ${ }^{2,14,15}$ but others may not. ${ }^{9,16,17}$ Musanje and Darvell ${ }^{9}$ reported that calculations using radiant exposure delivered to a restoration are based on the law of reciprocity, and the calculations do not recognize product behavior. Although this may be true, clinicians need usable guidelines when curing RBCs. A recent study showed that a better depth-of-cure comparison was achieved when a similar radiant exposure was delivered. ${ }^{18}$ In addition, most studies evaluating $\mathrm{RBC}$ polymerization used different irradiance and curing time combinations and different light sources at one curing distance. ${ }^{2,9,14-18}$ Determining curing protocols remains complicated because of variations in $\mathrm{RBCs}$ and the complexities of polymerization kinetics. The literature recommends that manufacturers provide curing protocols identifying the required energy for each RBC. ${ }^{9,14}$ Unfortunately, detailed curing protocols at clinically relevant distances to effectively activate polymerization are rarely provided, and clinicians are left to subjectively determine appropriate exposure times. Determining the curing effectiveness for different distances based on radiant exposure calculations from the irradiance and curing time provided by the manufacturer was worth investigating.

The aims of this study were 1) to investigate the influence of distance on the degree of conversion (DC) of an RBC when similar radiant exposure is achieved using multiple light-emitting-diode (LED) curing units and 2) to explore the correlation among irradiance, radiant exposure, and DC for multiple LED units at two clinically relevant distances.

\section{METHODS AND MATERIALS}

\section{Light Characterization}

Six LCUs were explored in this study (Table 1). The irradiance and spectral irradiance for each curing unit were measured using a managing accurate resin curing-resin calibrator (MARC-RC) system (BlueLight Analytics, Halifax, Canada). The system had custom-designed top and bottom 4-mm cosine corrector sensors designed to collect the light output at $180^{\circ}$ to eliminate any optical interference issues associated with the light-collection sampling geometry. ${ }^{19}$ The measurements collected from the top sensor represented the irradiance and radiant exposure received on the top RBC surface. The bottom sensor measurements obtained under an $\mathrm{RBC}$ increment in real time represented the irradiance and radiant exposure received on the bottom surfaces. $8,20-23$

The irradiance measurements and specimen preparation were performed in a constant-temperature room $\left(21^{\circ} \mathrm{C}\right)$ with $380-$ to $520-\mathrm{nm}$ ambient light filtered from the environment. On the top sensor, each curing unit position was standardized using a mechanical arm, with the guide tip centered over the sensor. Each unit was positioned at 0, 2, 4, 6, and 8 $\mathrm{mm}$ between the guide tip and the top sensor to collect the irradiance and spectral emission $(n=6 /$ LCU/distance). The collected irradiance was used to adjust the curing times for each tested distance to 
Table 1: Light-Curing Units (LCUs) Explored in the Study

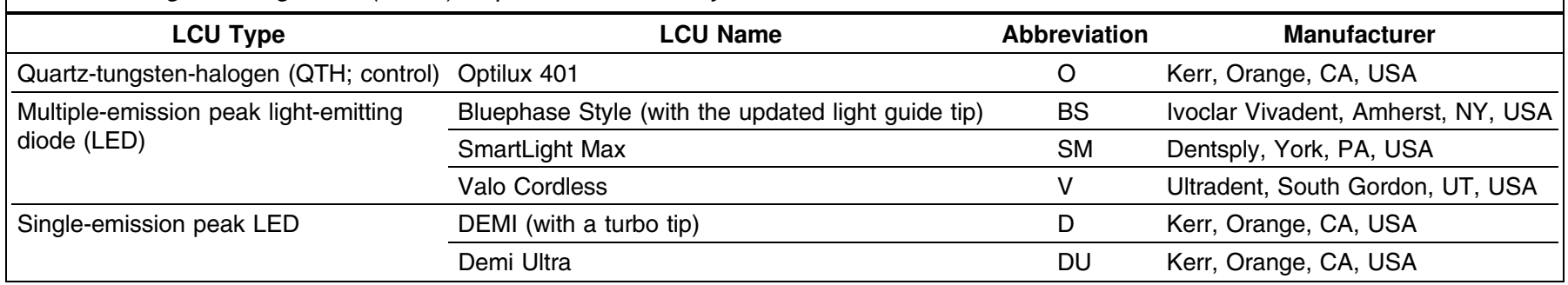

roughly maintain the radiant exposure at $10-11 \mathrm{~J} /$ $\mathrm{cm}^{2}$ on the top surface. This was consistent with manufacturer's irradiance and curing time guidelines for the dual-photoinitiator RBC (Tetric EvoCeram Bleaching shade XL, Ivoclar Vivadent, Amherst, NY, Lot No. T25427). Each LED unit was fully charged before collecting measurements, and the quartz-tungsten-halogen (QTH) unit fan was allowed to completely turn off between measurements. The RBC contained camphorquinone (CQ) and diphenyl (2,4,6-trimethylbenzoyl) phosphine oxide (TPO) photoinitiator systems, with less concentration of CQ compared with other Tetric EvoCeram shades. ${ }^{24}$ This particular RBC was selected with the intent of accentuating potential differences in polymerization using single- or multiple-emission peak LED units.

\section{Specimen Fabrication}

Each LCU was positioned over the bottom MARC-RC sensor in a setup similar to the top sensor. Square specimens $(5 \times 5 \times 2 \mathrm{~mm})$ were fabricated using a Delrin mold as described in a previous study $(n=3 /$ LCU/distance). ${ }^{22}$ The RBC was placed in the mold and sandwiched between two 0.002-mm-thick clear Mylar strips (Matrix Strips, DuPont MYLAR, Chester, VA) and glass slides to remove the excess material. The glass slides were removed, and the mold with Mylar strips was placed in the bottom sensor well. Each light guide tip was positioned at 2or 8-mm curing distance from the top specimen surface, to represent a good and worst clinical case scenario, respectively. ${ }^{3}$ Each curing unit was activated, and specimens were cured from the top using the adjusted curing times. The bottom sensor recorded the irradiance, radiant exposure, and spectral emission measurements passing through the 2-mm-thick specimens. The Mylar strip placed on the top specimen surfaces absorbed 3.5\%-6.3\% of the LCUs' irradiation. This absorbance was similar to another study. ${ }^{25}$ The Mylar strips were removed, and the specimens were placed in a container, then wrapped with aluminum foil to prevent specimen exposure to light. Specimens were stored dry in a $37^{\circ} \mathrm{C}$ incubator for 24 hours. ${ }^{26}$

\section{Measurements}

The top and bottom specimen surfaces were finished using a Struers Rotopol 4 polishing unit with 1200-, 2400-, and 4000-grit SiC abrasive paper and polished using a 1- $\mu \mathrm{m}$ alcohol-based diamond polishing suspension (Struers, Ballerup, Denmark) to produce a flat and smooth surface. Specimens were ultrasonically cleansed for 20 minutes in deionized water to produce clean surfaces for DC testing. The DC measurements were collected using micro-Raman spectroscopy (FORAM, CRAIC Technologies, San Dimas, CA, USA) with a 785-nm laser excitation. Five scans for each spectrum measurement were collected and processed with FORAM PC software. Spectra of the uncured and cured RBC were recorded $(\mathrm{n}=3)$. The fourth day after specimen preparation, DC measurements were collected from the top and bottom surfaces (50/surface) in standardized locations of a $3 \times 3-\mathrm{mm}$ checkerboard grid pattern. The measurements obtained were at least $1-\mathrm{mm}$ away from all edges of the specimen. The DC calculation was attained by comparing the relative change of the vinyl $\mathrm{C}=\mathrm{C}$ band peak height $\left(1640 \mathrm{~cm}^{-1}\right)$ before and after the polymerization and the aromatic $\mathrm{C}=\mathrm{C}$ reference band peak height $\left(1610 \mathrm{~cm}^{-1}\right)$ using the following equation ${ }^{27}$ :

$$
\mathrm{DC} \%=\left(1-\frac{\text { cured }\left(\frac{\text { peak height at } 1640}{\text { peak height at 1610 }}\right)}{\text { uncured }\left(\frac{\text { peak height at } 1640}{\text { peak height at 1610 }}\right)}\right) \times 100
$$

\section{Statistical Analysis}

The effects of the LCUs and distance from the specimen $(2$ or $8 \mathrm{~mm}$ ) on irradiance, radiant exposure, and DC were analyzed using multipleway analysis of variance (ANOVA), with each LCUdistance combination allowed to have a different variance. Pearson correlation coefficients were cal- 
culated to evaluate the linear associations among irradiance, radiant exposure, and DC. The percentage decrease in irradiance, radiant exposure, and DC between the top and bottom surfaces for each LCU at each curing distance was calculated using a Student $t$-test. A 5\% significance level was used for all tests. The normality assumptions were assessed and met for the ANOVA.

\section{RESULTS}

\section{Irradiance and Spectral Irradiance Measurements}

Figure 1 shows an inverse relationship between irradiance and curing distance in a distinct pattern for each LCU. Figure 2 displays a positive relationship between curing distance and curing time to achieve a similar radiant exposure, which was unique for each curing unit. When curing with Bluephase Style (BS), a relatively equivalent irradiance and curing time were displayed for up to $6 \mathrm{~mm}$ from the top sensor. On the other hand, when the distance was increased, the remaining LCUs revealed a relatively gradual increase in curing time and decrease in irradiance. Table 2 depicts that curing with Optilux $401(\mathrm{O})$ and BS showed the least decrease in irradiance and the least increase in the required curing time between 2 - and $8-\mathrm{mm}$ distances to achieve the targeted radiant exposure. On the other hand, SmartLight Max (SM), Valo Cordless (V), and Demi Ultra (DU) showed the most decrease in irradiance and the most increase in the required curing time.

Figure 3 displays the spectral irradiance of the curing units combined with the spectral absorption of CQ and TPO photoinitiators at 2-mm distance. Figure 3 a shows a broad spectral irradiance curve for the QTH unit that fell within the absorption ranges of CQ (peaks approximately at $470 \mathrm{~nm}$ ) and TPO (peaks approximately at $380 \mathrm{~nm}$ ). The multiple emission peak LED units emitted two spectral peaks, one in the blue range $(420-520 \mathrm{~nm})$ and one in the violet range $(380-425 \mathrm{~nm})$, which fell within the absorption range of $\mathrm{CQ}$ and TPO, respectively. Both the QTH and multiple-emission peak LED units had higher output in the $\mathrm{CQ}$ range than the TPO range. However, the multiple-emission peak LED units had a higher output than the QTH unit in the CQ and TPO ranges. The single-emission peak LED units had one spectral peak in the blue range that fell within the maximum absorption range of the CQ. Each LCU exhibited a unique peak wavelength and peak height. Furthermore, Figure $3 \mathrm{~b}$ shows that the spectral irradiance dramatically

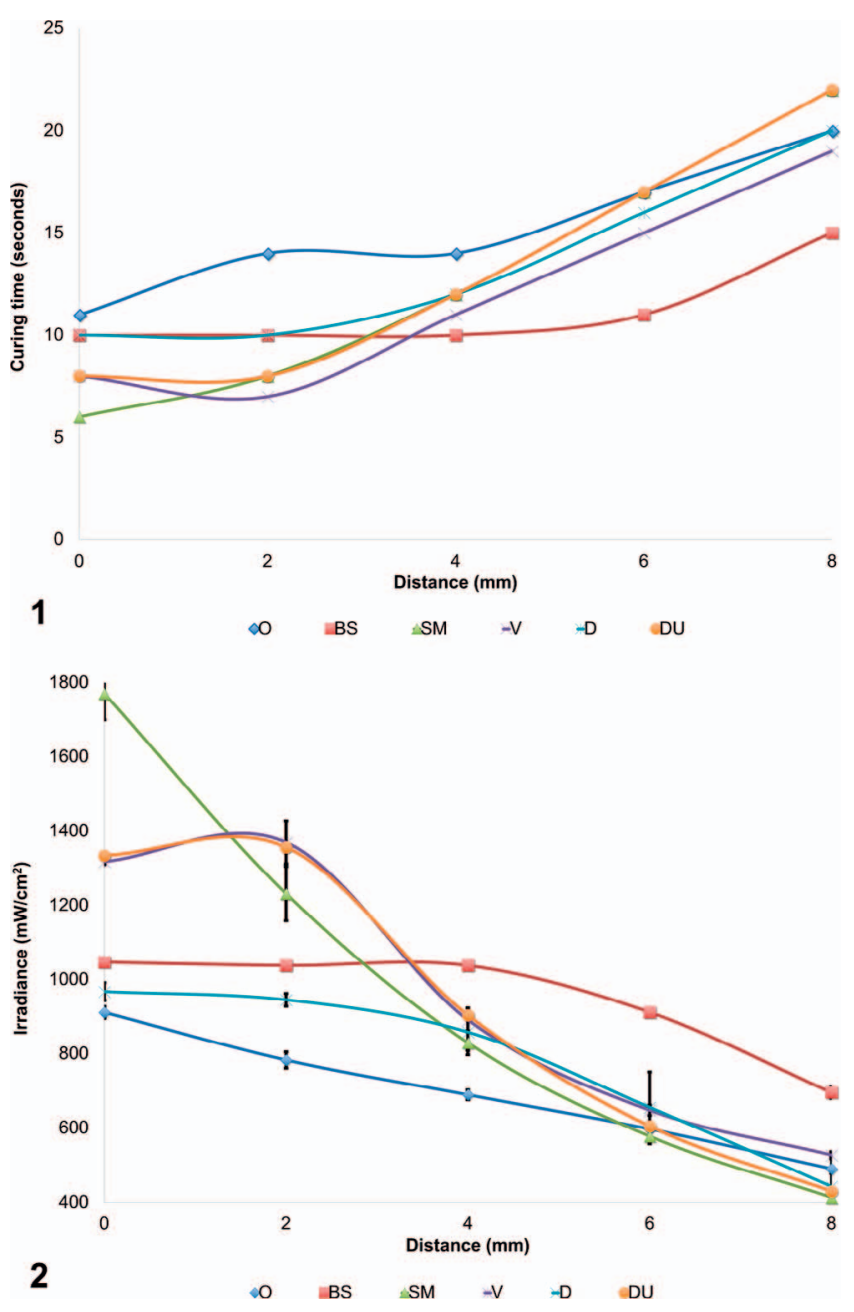

Figure 1. Mean $(S D)$ irradiance $\left(\mathrm{mW} / \mathrm{cm}^{2}\right)$ measurements at multiple distances $(\mathrm{mm})$ for the LCUs collected using the MARC-RC top sensor. The irradiance decreased with the increase in the distance in a pattern that was unique for each LCU.

Figure 2. Curing time (seconds) needed to maintain the radiant exposure (10-11 $\left.\mathrm{J} / \mathrm{cm}^{2}\right)$ at multiple curing distances $(\mathrm{mm})$ for each $L C U$ using the MARC-RC top sensor. The curing times were adjusted by maintaining the radiant exposure at 0-, 2-, 4-, 6-, and 8-mm distances based on the manufacturer's information to polymerize Tetric EvoCeram bleaching shade $X L R B C$. The curing times increased with the increase in the distance in a pattern that was unique for each LCU.

decreased on the bottom surfaces of the 2-mm-thick specimens compared with the top. Also, the bottom sensor no longer effectively detected the violet curves when using the multiple-emission peak LED lights. Similar trends were observed at 8-mm distances.

\section{Irradiance, Radiant Exposure, and DC Measurements}

Figure 4 shows the comparison of irradiances received on the top and bottom surfaces among the LCUs, where significant differences were detected 


\begin{tabular}{|lcc|}
\hline Table 2: & \multicolumn{2}{c|}{$\begin{array}{c}\text { Percentage Decrease in Irradiance and } \\
\text { Percentage Increase in Curing Time to Maintain } \\
\text { a 10-11 } \mathrm{J} / \mathrm{cm}^{2}\end{array}$} \\
\hline LCU & $\begin{array}{c}\text { Irradiance, \% } \\
\text { Decrease Between } \\
\text { 2- and 8-mm Distances }\end{array}$ & $\begin{array}{c}\text { Curing Time, \% } \\
\text { Increase Between } \\
\text { 2- and 8-mm Distances }\end{array}$ \\
\hline O & 37 & 43 \\
\hline BS & 33 & 50 \\
\hline SM & 67 & 175 \\
\hline V & 62 & 171 \\
\hline D & 53 & 100 \\
\hline DU & 68 & 175 \\
\hline
\end{tabular}

regardless of the surface or curing distance. The irradiance was significantly higher at 2-mm than 8$\mathrm{mm}$ curing distance for all LCUs, regardless of surface. Generally, the trend of significant differences in irradiance among the LCUs for the top was not the same for the bottom, regardless of the curing distance. On the top (Figure 4a), the irradiance values at $2-\mathrm{mm}$ distance revealed that $\mathrm{V}$ was significantly higher than the remaining LCUs, with the lowest values seen when curing with O. At $8 \mathrm{~mm}$, the top surface values for BS were significantly higher than the remaining units, and DEMI (D) was significantly lower. On the bottom surfaces (Figure $4 \mathrm{~b}), \mathrm{SM}$ and DU at 2-mm curing distance showed significantly higher irradiance, and $\mathrm{O}$ had the lowest values. At $8 \mathrm{~mm}$, BS was significantly higher than the remaining lights, and SM and D were significantly lower. The irradiance values decreased significantly between the top and bottom surfaces irrespective of the original values received on the top.

The radiant exposure comparison among the LCUs at each distance showed values that were significantly higher on the top than the bottom, regardless of the curing distance or LCU (Table 3). The values significantly decreased between $85.6 \%$ $92.6 \%$ on the bottom regardless of the original values received on the top. On the bottom surfaces, radiant exposure was significantly lower at $2 \mathrm{~mm}$ than 8 $\mathrm{mm}$, except when using $\mathrm{O}$.

On the top surfaces, the DC value at $2-\mathrm{mm}$ distance was significantly higher when using SM than when using the QTH or single-emission peak LED units (Figure 5a). Also, using the singleemission peak LED units resulted in significantly lower DC values than the remaining LCUs, except that DU did not show significant differences from $\mathrm{BS}$. At the 8-mm curing distance, $\mathrm{O}$ and $\mathrm{BS}$ showed significantly higher top surface DC values than the
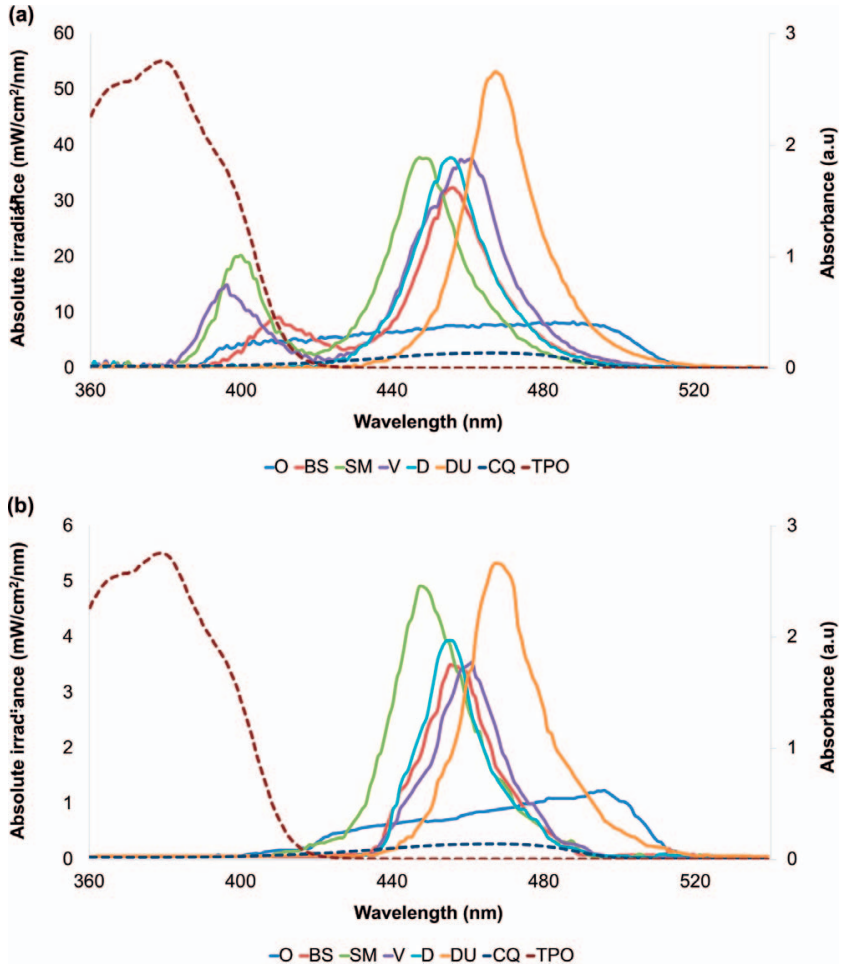

Figure 3. Representative spectral irradiance curve of the LCUs collected using the top and bottom MARC-RC sensors combined with the measured absorbance spectrum of CQ and TPO photoinitiators at $0.005 \mathrm{M}$ concentration. (a) Spectral irradiance curve at 2-mm distance from the top MARC-RC sensor, representing the irradiance received on the top surfaces. The spectral irradiance of the longer-wavelength curve encompasses the CQ photoinitiator absorption range. The shorter wavelength curve for the QTH and multiple-emission peak LED units encompasses the TPO photoinitiator absorption range. (b) Spectral irradiance curve at 2-mm distance from the top specimen surfaces passing through the 2-mm increment and detected by the bottom MARC-RC sensor, representing the irradiance received on the bottom surfaces. The spectral irradiance dramatically decreased on the bottom. The spectral irradiance of the shorter-wavelength curves was no longer detected by the bottom sensor when using the multipleemission peak $L E D$ units.

single-emission peak LED units. Using DU revealed significantly lower DC values than the remaining LCUs, except for D. At $2 \mathrm{~mm}$, D had significantly lower bottom surface values than $\mathrm{O}, \mathrm{BS}$, and $\mathrm{SM}$ (Figure $5 \mathrm{~b}$ ). At $8 \mathrm{~mm}, \mathrm{~V}$ had significantly higher DC on the bottom than SM and D. In addition, D had significantly higher DC than SM. The DC values were significantly higher at $2-\mathrm{mm}$ than $8-\mathrm{mm}$ distance when using SM regardless of the surface (Figure 6).

The percentage decrease in the $\mathrm{DC}$ values between the top and bottom surfaces ranged from $0.2 \%-7.4 \%$ and was significant at 2-mm distance when using the QTH or multiple-emission peak LED lights (Figure 7). At $8 \mathrm{~mm}, \mathrm{O}$ and $\mathrm{SM}$ showed a significant decrease in $\mathrm{DC}$ values from the top to the bottom surfaces. 
(a)

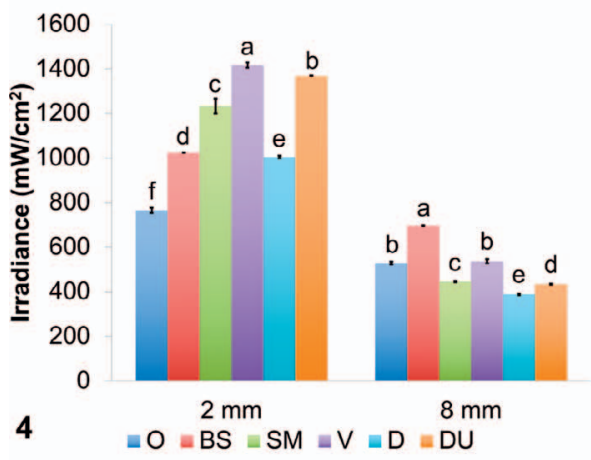

(a)

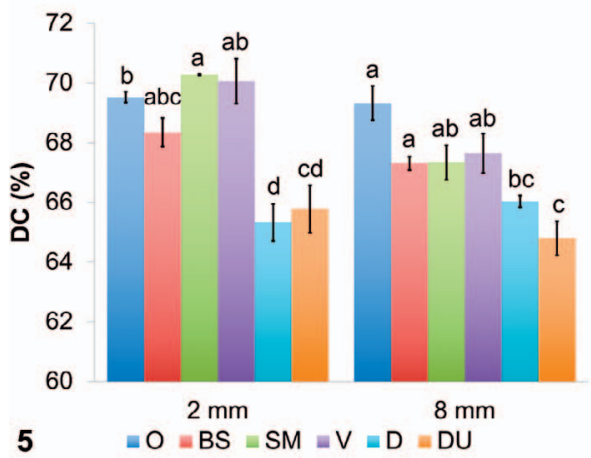

(a)

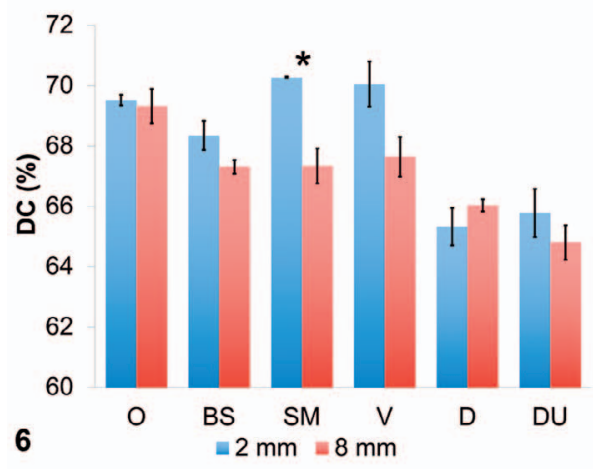

(b)

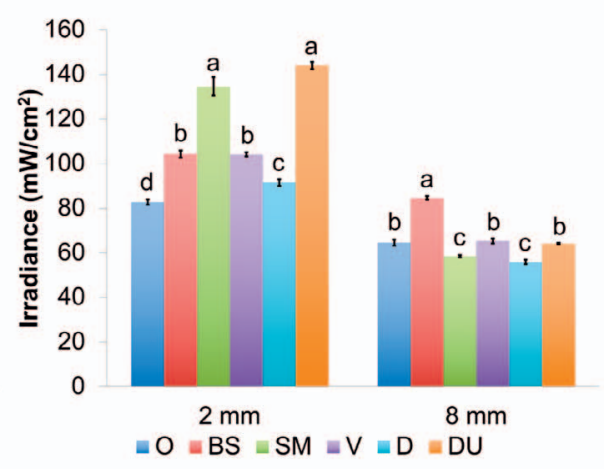

(b)

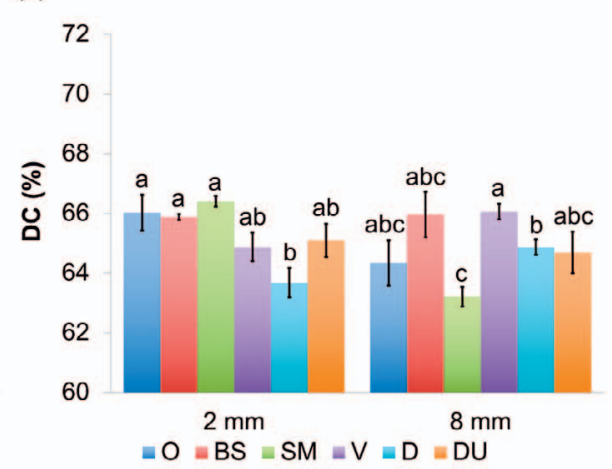

(b)

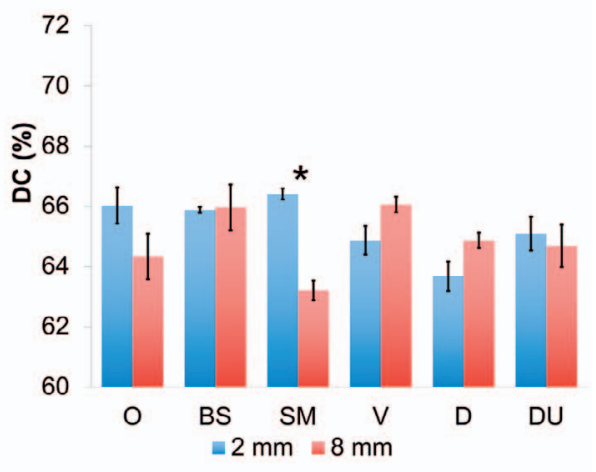

Figure 4. Mean (SE) irradiance $\left(\mathrm{mW} / \mathrm{cm}^{2}\right)$ values received by the $R B C$ specimens at 2- and 8- $\mathrm{mm}$ distances. (a) Mean irradiance on the top surfaces. (b) Mean irradiance on the bottom surfaces. The irradiance measurements were collected from a MARC-RC system. The top $M A R C-R C$ sensor recorded the irradiance values representing those received on the top specimen surfaces at 2- and 8-mm distances. The bottom sensor detected the irradiance values passing through the 2-mmthick RBC increment at 2- and 8-mm distances. Letters represent significant differences among the LCUs at each distance for each surface. For each LCU, the irradiance values on the top for each LCU was significantly higher than the bottom, regardless of the distance. $4 b$ was smoothed for clarity.

Figure 5. Mean (SE) DC (\%) values for RBC specimens at 2- and 8- $\mathrm{mm}$ distances. (a) Mean DC values on the top surfaces. (b) Mean DC values on the bottom surfaces. The DC measurements were collected using micro-Raman spectroscopy. Letters represent significant differences among the LCUs at each curing distance for each surface.

Figure 6. Mean (SE) DC (\%) values for RBC specimens at 2- and 8-mm distances. (a) Mean DC values on the top surfaces. (b) Mean $D C$ values on the bottom surfaces. The DC measurements were collected using micro-Raman spectroscopy. The asterisk represents significant differences between the 2- and 8-mm distance for each $L C U$ on each surface.

Figure 7. Mean (SE) DC (\%) values on the top and bottom surfaces of the specimens at 2- and 8-mm distances. The asterisk represents significant differences between the top and bottom surfaces for each LCU.

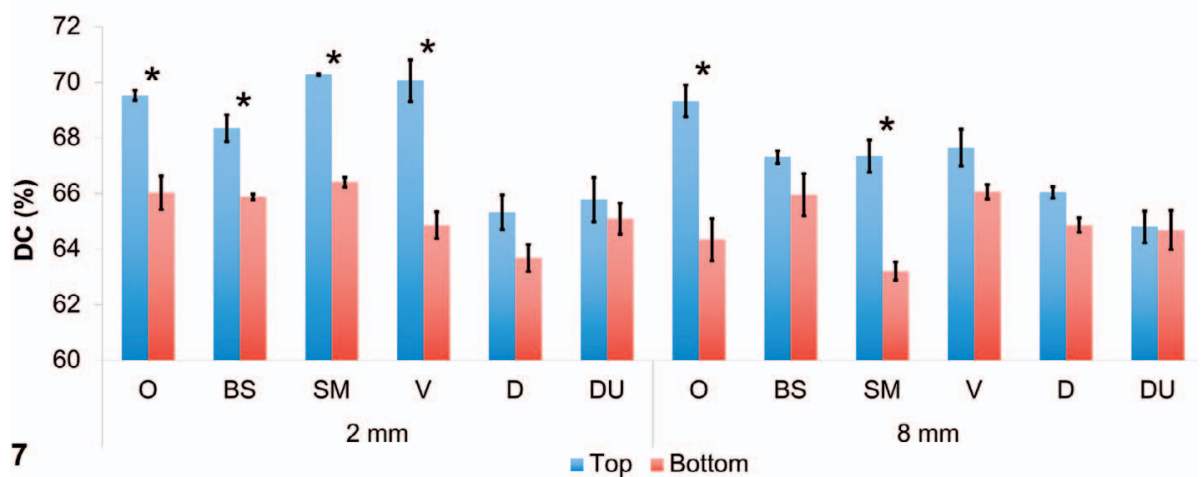




\begin{tabular}{|c|c|c|c|}
\hline Table 3: & $\begin{array}{l}\text { Mean (SE) } \\
\text { on the Top }\end{array}$ & $\begin{array}{l}\text { ant Exposure } \\
\text { Bottom Spec }\end{array}$ & $\begin{array}{l}\text { Received } \\
\text { urfaces }^{a}\end{array}$ \\
\hline Distance, & LCU & Radiant & $\mathrm{re}, \mathrm{J} / \mathrm{cm}^{2}$ \\
\hline & & Top & Bottom \\
\hline 2 & 0 & $10.63(0.16)$ & $1.15(0.017)$ \\
\hline & BS & $10.34(0.04)$ & $1.05(0.019)$ \\
\hline & SM & $9.72(0.24)$ & $1.07(0.022)$ \\
\hline & $\mathrm{V}$ & $10.06(0.04)$ & $0.74(0.007)$ \\
\hline & $\mathrm{D}$ & $10.26(0.05)$ & $0.93(0.016)$ \\
\hline & DU & $10.54(0.10)$ & $1.11(0.016)$ \\
\hline 8 & $\mathrm{O}$ & $10.08(0.13)$ & $1.15(0.037)$ \\
\hline & BS & $10.29(0.21)$ & $1.26(0.015)$ \\
\hline & SM & $9.89(0.08)$ & $1.30(0.017)$ \\
\hline & $\mathrm{V}$ & $10.81(0.19)$ & $1.29(0.029)$ \\
\hline & $\mathrm{D}$ & $10.44(0.10)$ & $1.51(0.031)$ \\
\hline & DU & $9.83(0.05)$ & $1.43(0.015)$ \\
\hline $\begin{array}{l}{ }^{a} \text { The radian } \\
\text { system. The } \\
\text { received on } \\
\text { bottom senso } \\
m m R B C \text { inc } \\
L C U, \text { the rac } \\
\text { higher than }\end{array}$ & $\begin{array}{l}\text { the top spec } \\
\text { sor detected th } \\
\text { crement at 2- } \\
\text { adiant exposur } \\
\text { on the bottom }\end{array}$ & $\begin{array}{l}\text { ents were colle } \\
\text { recorded the } \\
\text { faces at 2- an } \\
\text { exposure value } \\
\text { 7 distances. At } \\
\text { on the top sur }\end{array}$ & $\begin{array}{l}\text { a MARC-RC } \\
\text { posure values } \\
\text { listances. The } \\
\text { through the 2- } \\
\text { tance for each } \\
\text { re significantly }\end{array}$ \\
\hline
\end{tabular}

The ANOVA revealed that the interaction between the LCU and curing distance had a significant effect on the irradiance and radiant exposure received on the top and bottom surfaces and on the DC values on the bottom surfaces.

\section{Correlation Among Irradiance, Radiant Exposure, and DC}

An overall strong positive correlation between irradiance and radiant exposure was detected (0.87-1) regardless of the curing distance, with a few exceptions: on the top surfaces at $2 \mathrm{~mm}$ distance, a moderate positive correlation was detected (0.57) when $\mathrm{V}$ was used, and a negative correlation was shown $(-0.73$ and -1$)$ when $O$ and DU were used, respectively. On the top surfaces of $8-\mathrm{mm}$ distance, a weak positive correlation was detected $(0.14$ and 0.13 ) when D and DU were used, respectively. A moderate correlation was found between DC and irradiance and between DC and radiant exposure regardless of distance. However, the correlations varied for each LCU, at each distance, and on each surface (data not shown).

\section{DISCUSSION}

\section{Influence of Distance on Irradiance}

The outcomes of this study showed an inverse relationship between the curing distance and irradiance, resulting in a direct relationship between curing time and distance to maintain a similar radiant exposure (Figures 1 and 2). However, the pattern was unique for each LCU. The percentage change in irradiance and curing time between 2 - and 8-mm distances was the least for $\mathrm{O}$ and BS (Table 2). A more uniform spectral radiant power has been reported for the QTH and from the BS's updated guide tip than with the remaining LED units. ${ }^{22}$ On the other hand, relatively similar patterns of change were seen when using SM, V, and DU, requiring the greatest percentage increase. Those three curing units had their LED chips located within the light guide head and not the body. This may suggest that irradiance values at different distances are affected by the location of the LED chips within the curing unit. The overall results agree with previous studies that reported that mean irradiance values for a given location are negatively affected by increased curing distance as emitted light diverges over a larger surface area. . $^{3,8,28,29}$

\section{Spectral Irradiance Received on the Specimen Surfaces}

The outcomes showed variations in spectral irradiance between the QTH and LED units, in addition to differences among the LED units (Figure 3). This agreed with similar studies. ${ }^{1,30}$ Interestingly, spectral irradiance showed a large drop from the top to the bottom, and the peak heights varied for each LED unit on each surface. These differences may be attributed to the LCU type. A QTH unit has a wide spectral emission curve $(390-520 \mathrm{~nm})$, which includes the wavelengths needed by most photoinitiators. ${ }^{4,31}$ However, a QTH unit may not activate TPO as effectively as CQ because it has a lower spectral irradiance in the TPO adsorption region compared with the $\mathrm{CQ}$ region. A multiple-emission peak LED unit emits light within narrow spectral ranges that fall within the maximum absorption range of $\mathrm{CQ}$ $(450-470 \mathrm{~nm})$ and TPO $(380-420 \mathrm{~nm}){ }^{4,31}$ The outcomes agree with the literature. ${ }^{1,4,31}$ The LED units may provide equivalent polymerization in a shorter curing time because more photons are being provided in more effective absorption ranges., 41 This was relatively supported by our study, in which the QTH unit needed more curing time at 2- and 8$\mathrm{mm}$ distances compared with the LED units.

The spectral irradiance peaks in the shorter wavelength region on the bottom surfaces were not effectively detected compared with the top, regardless of the LED unit used. These observations may be partially explained by the extent of light transmission through the specimens ${ }^{23,32,33}$ : the shorter 
wavelength becomes more scattered or refracted compared with the longer wavelengths. These findings were in agreement with previous work and the results of other authors. ${ }^{22,34}$ Also, photoinitiators, pigments, and filler particles may have hampered light transmission through the specimens by absorbing, scattering, or refracting the light at the resin-filler interface. ${ }^{2,10}$

\section{Influence of Distance on Irradiance, Radiant Exposure, and DC Measurements on Specimen Surfaces}

Comparisons of the mean irradiance values among the LCUs showed significant differences regardless of the distance and surface (Figure 4). It was intriguing to see that the curing unit that exhibited higher irradiance values at 2 -mm distance did not necessarily provide a higher irradiance at $8-\mathrm{mm}$ distance. Similarly, the unit that exhibited higher irradiance values on the top did not necessarily show higher irradiance on the bottom. Since the curing unit position was standardized, the overall observations were highly influenced by the differences in light sources. ${ }^{4,31}$ An LCU's effectiveness depends on the radiant power and photons it emits that activate the photoinitiators within an RBC. ${ }^{35}$ For $\mathrm{D}$, the significantly lower mean irradiance values detected at 8-mm curing distance compared with the remaining curing units could be attributed to the Turbo light guide tip, which has an exit diameter smaller than the entry diameter, producing a wider light cone as the distance increases. ${ }^{28}$ Consequently, irradiance values are negatively affected because less radiant power strikes any delineated portion of the surface with increasing distance. It is important to note that the measurements reported from the sensors do not reflect the irradiance of the entire light guide tip but only of the guide tip area that fell over the sensor. In this study, the measurements collected were from the center of each guide tip, and the DC measurements obtained from the specimens relatively coincided with the sensor dimensions. Thereby, the irradiance and radiant exposure values recorded reflected the irradiance and radiant exposure received on the top and bottom specimen surfaces.

Comparisons of the irradiance and radiant exposure between the top and bottom surfaces showed that the irradiance transmitted through the specimen and radiant exposure detected on the bottom significantly decreased compared with the top (Figure 4; Table 3). Interestingly, the irradiance and radiant exposure decreased between $85.2 \%$ -
92.6\% from the top to the bottom. This suggests that the light from the LCUs may have transmitted through the specimens in a similar manner, regardless of the distance or irradiance values on the top. The nonsignificant difference in the mean radiant exposure values on the bottom was consistent with similar radiant exposure provided on the top. The results support previous work. ${ }^{22}$

Sufficient conversion of monomer into polymer during RBC polymerization is essential to produce an RBC with satisfactory properties. ${ }^{2,36}$ The DC measurement may estimate the properties of the final restoration because a correlation was suggested between DC and microhardness, elastic modulus, glass transition temperature, wear, marginal breakdown, elutable substances from the composite, and volumetric shrinkage. ${ }^{13,36-43}$ The clinical performance of an RBC may be predicted based on the DC values, in which higher DC values may predict higher mechanical and physical properties. Assessment of the bottom/top hardness ratio is used as an indicator of the curing effectiveness of the RBC. A bottom/top hardness ratio of $80 \%$ is commonly used as the minimally acceptable ratio for RBCs. ${ }^{23,44}$

The DC results showed several interesting findings. First, similar DC values were achieved with similar radiant exposure, although some variation in DC values occurred regardless of the LCU, distance, and surface (Figure 5). This was true even with significant irradiance reduction on the bottom surface and with increased distance (Figure 4).

Second, at each distance, significant differences in DC values were observed among the LCUs. It was intriguing that the unit that showed a higher irradiance value did not necessarily display higher DC values and vice versa, regardless of the surface or curing distance (Figures 4 and 5). DC values of 50\%-75\% are typically achieved when using a conventional RBC. ${ }^{45}$ In our study, all LCUs resulted in DC values greater than $63 \%$, regardless of the surface or curing distance. Therefore, our results indicated that sufficient polymerization may be achieved when radiant exposure values between 0.7-1.5 J/cm ${ }^{2}$ and irradiance values between 55.9$84.6 \mathrm{~mW} / \mathrm{cm}^{2}$ are received on the bottom. These findings were in agreement with those of another study. ${ }^{19,22}$

Interestingly, although using the single-emission peak LED units exhibited the lowest DC values on the top among the LCUs, the same was not true on the bottom. This suggests that light was not attenuated to a degree that compromised polymeri- 
zation on the bottom. Our results agreed with those of another study that explored various LCUs ${ }^{46}$ and agreed with a study that evaluated bulk-filled RBCs and concluded that light transmission did not alter polymerization kinetics and that DC cannot be related to light transmittance. ${ }^{47}$ Another possible explanation for the higher DC values on the bottom is the reflection of the light off the radiopaque bottom sensor. The findings agreed with other studies, which suggested that a similar degree of polymerization could be achieved when using a QTH or single- or multiple-emission peak LED units. ${ }^{48,49}$

Third, when comparing the 2- and 8-mm distances for each LCU, it was revealed that distance did not have a significant influence on DC values, except for SM (Figure 6). The significant differences among the LCUs at each distance may also be explained by the differences in the units and the amount of photons received by the specimens. The nonsignificant differences between the 2- and 8-mm distances for each LCU may be attributable to the similar radiant exposure values received by the specimens allowing for sufficient generation of free radical growth centers and satisfactory polymerization. ${ }^{1,25}$ In addition, the prolonged curing time at the 8 -mm distance may have increased heat generation favoring polymerization. $^{2,50}$ The significantly higher mean DC values for $\mathrm{SM}$ at 2 -mm than 8 -mm distance regardless of the surface may be due to the angled position of the blue and violet LED chips within the unit head. This may have allowed the emitted light to be more focused at $2 \mathrm{~mm}$ than $8 \mathrm{~mm}$. The number of photons hitting the specimens may have impacting the amount of photoinitiators activated and the rate of polymerization.

Finally, the percentage decrease in DC values from the top to bottom was significantly higher with the QTH and multiple-emission peak LED units at $2-\mathrm{mm}$ distance and with $\mathrm{O}$ and $\mathrm{SM}$ at $8-\mathrm{mm}$ distance (Figure 7). At 2-mm distance, the significantly higher DC values on the top when using the QTH and multiple-emission peak LED units may be because TPO was effectively activated on the top but not at the deeper parts of the specimen compared with $\mathrm{CQ} .{ }^{20}$ In addition, this suggests that TPO was not effectively activated when using the single-emission peak LED units due to the absence of the violet LED chip. Nevertheless, polymerization was not compromised when using the singleemission peak LED units, which may indicate sufficient free radicals were generated, allowing satisfactory polymerization activation. However, at 8-mm distance, the significant differences in DC percentage decrease when using $\mathrm{O}$ and SM may also be partially explained by the amount of photoinitiator activation and complexity of polymerization kinetics.

The ANOVA outcomes showed that interaction between the LCU and distance had a significant effect on irradiance and radiant exposure received on the top and bottom surfaces and on the DC values on the bottom. This may be explained by the differences among the LCUs, light transmission through the specimens, and complexity of polymerization kinetics, as mentioned. Nevertheless, it is important to note that the observations in this study may translate to RBCs with similar composition, shade, and translucency, but the findings may differ to some extent when using different RBCs.

\section{Correlation Among Irradiance, Radiant Exposure, and DC}

The correlation outcomes demonstrated that the strength of the correlation among the average irradiance, radiant exposure, and DC values on the top and bottom surfaces was dependent on LCU and curing distance. Although a generally positive, strong correlation between the average irradiance and radiant exposure values existed, this did not necessarily result in a strong correlation between the average irradiance and DC values nor between radiant exposure and DC, regardless of the LCU used, curing distance, or specimen surface.

\section{Clinical Impact}

The findings are of clinical value because they show the relationship between distance and curing time. Using the multiple-emission peak curing unit, BS can be convenient for clinicians because the manufacturer's recommended curing time may be used up to a $6-\mathrm{mm}$ distance from the restoration. When increasing to an 8-mm distance between the guide tip and cavity floor, it was necessary to almost double the curing times when using a QTH or a single-emission peak curing unit that had the LED chip in the body of the unit. The curing time was tripled when using the curing unit that had the LED chips within the unit's head.

For all units, obtaining similar radiant exposure resulted in satisfactory polymerization. Although not an exact predictor, radiant exposure received by the restoration was an effective guide for polymerization. This may be a helpful guide for clinicians when specific curing instructions are not provided for clinically relevant distances. 
There is potential benefit for manufacturers to provide curing protocol guides for a given RBC using different LCUs at multiple distances. Further investigation is needed to explore the influence of these distances on RBC mechanical properties.

\section{CONCLUSIONS}

1. Achieving similar radiant exposure with increased curing distance using the LCUs investigated resulted in satisfactory polymerization on the top and bottom surfaces of the RBC explored with respect to $\mathrm{DC}$, although the same $\mathrm{DC}$ values were not reached.

2. Sufficient polymerization was achieved when the bottom RBC surfaces received radiant exposure values between $0.7-1.5 \mathrm{~J} / \mathrm{cm}^{2}$ and irradiance values between $56-85 \mathrm{~mW} / \mathrm{cm}^{2}$.

3. A moderate correlation existed among irradiance, radiant exposure, and DC.

\section{Acknowledgements}

This work was part of $\mathrm{Dr}$ Al-Zain's $\mathrm{PhD}$ project. The scholarship support for Dr Al-Zain from King Abdulaziz University Faculty of Dentistry, Jeddah, Saudi Arabia, is acknowledged.

\section{Conflict of Interest}

The authors of this article certify that they have no proprietary, financial, or other personal interest of any nature or kind in any product, service, and/or company that is presented in this article.

\section{(Accepted 8 November 2018)}

\section{REFERENCES}

1. Price RB, Ferracane JL, \& Shortall AC (2015) Lightcuring units: a review of what we need to know Journal of Dental Research 94(9) 1179-1186. doi:10.1177/ 0022034515594786

2. Leprince JG, Palin WM, Hadis MA, Devaux J, \& Leloup G (2013) Progress in dimethacrylate-based dental composite technology and curing efficiency Dental Materials 29(2) 139-156. doi:10.1016/j.dental.2012.11.005

3. Price RB, Labrie D, Whalen JM, \& Felix CM (2011) Effect of distance on irradiance and beam homogeneity from 4 light-emitting diode curing units Journal of the Canadian Dental Association $\mathbf{7 7}$ b9.

4. Rueggeberg FA (2011) State-of-the-art: dental photocuring-a review Dental Materials 27(1) 39-52. doi:10.1016/j. dental.2010.10.021

5. Shortall AC, Price RB, MacKenzie L, \& Burke FJ (2016) Guidelines for the selection, use, and maintenance of LED light-curing units-part 1 Brazilian Dental Journal 221(8) 453-460. doi:10.1038/sj.bdj.2016.772
6. Platt JA \& Price RB (2014) Light curing explored in Halifax Operative Dentistry 39(6) 561-563. doi:10.2341/ 1559-2863-39.6.561

7. Shortall AC, Price RB, MacKenzie L, \& Burke FJ (2016) Guidelines for the selection, use, and maintenance of LED light-curing units-part II Brazilian Dental Journal 221(9) 551-554. doi:10.1038/sj.bdj.2016.814

8. Beolchi RS, Moura-Netto C, Palo RM, Rocha Gomes Torres C, \& Pelissier B (2015) Changes in irradiance and energy density in relation to different curing distances Brazilian Oral Research 29. doi:10.1590/ 1807-3107BOR-2015.vol29.0060

9. Musanje L \& Darvell BW (2003) Polymerization of resin composite restorative materials: exposure reciprocity Dental Materials 19(6) 531-541.

10. AlShaafi MM (2017) Factors affecting polymerization of resin-based composites: a literature review Saudi Dental Journal 29(2) 48-58. doi:10.1016/j.sdentj.2017.01.002

11. Felix CA \& Price RB (2003) The effect of distance from light source on light intensity from curing lights Journal of Adhesive Dentistry 5(4) 283-291.

12. Knezevic A, Zeljezic D, Kopjar N, \& Tarle Z (2008) Cytotoxicity of composite materials polymerized with LED curing units Operative Dentistry 33(1) 23-30. doi: 10.2341/07-16

13. Durner J, Obermaier J, Draenert M, \& Ilie N (2012) Correlation of the degree of conversion with the amount of elutable substances in nano-hybrid dental composites Dental Materials 28(11) 1146-1153. doi:10.1016/j.dental. 2012.08.006

14. Leprince JG, Hadis M, Shortall AC, Ferracane JL, Devaux J, Leloup G, \& Palin WM (2011) Photoinitiator type and applicability of exposure reciprocity law in filled and unfilled photoactive resins Dental Materials 27(2) 157-164. doi:10.1016/j.dental.2010.09.011

15. Feng L \& Suh BI (2007) Exposure reciprocity law in photopolymerization of multi-functional acrylates and methacrylates Macromolecular Chemistry and Physics 208(3) 295-306. doi:10.1002/macp.200600480

16. Selig D, Haenel T, Hausnerova B, Moeginger B, Labrie D, Sullivan B, \& Price RB (2015) Examining exposure reciprocity in a resin based composite using high irradiance levels and real-time degree of conversion values Dental Materials 31(5) 583-593. doi:10.1016/j. dental.2015.02.010

17. Wydra JW, Cramer NB, Stansbury JW, \& Bowman CN (2014) The reciprocity law concerning light dose relationships applied to BisGMA/TEGDMA photopolymers: theoretical analysis and experimental characterization Dental Materials 30(6) 605-612. doi:10.1016/j.dental.2014.02. 021

18. Erickson RL \& Barkmeier WW (2017) Effect of mold diameter on the depth of cure of a resin-based composite material European Journal of Oral Sciences 125(1) 88-92. doi:10.1111/eos.12325

19. Bucuta S \& Ilie N (2014) Light transmittance and micromechanical properties of bulk fill vs. conventional resin based composites Clinical Oral Investigations 18(8) 1991-2000. doi:10.1007/s00784-013-1177-y 
20. Arikawa H, Fujii K, Kanie T, \& Inoue K (1998) Light transmittance characteristics of light-cured composite resins Dental Materials 14(6) 405-411.

21. Eshmawi YT, Al-Zain AO, Eckert GJ, \& Platt JA (2018) Variation in composite degree of conversion and microflexural strength for different curing lights and surface locations Journal of the American Dental Association 149(10) 893-902. doi:10.1016/j.adaj.2018.06.004

22. Al-Zain AO, Eckert GJ, Lukic H, Megremis SJ, \& Platt JA (2018) Degree of conversion and cross-link density within a resin-matrix composite Journal of Biomedical Material Research part B Applied Biomaterials 106(4) 1496-1504. doi:10.1002/jbm.b.33960

23. Maghaireh GA, Price RB, Abdo N, Taha NA, \& Alzraikat $H$ (2018) Effect of thickness on light transmission and vickers hardness of five bulk-fill resin-based composites using polywave and single-peak light-emitting diode curing lights Operative Dentistry June 28 Epub ahead of print. doi:10.2341/17-163-L

24. Palin WM, Senyilmaz DP, Marquis PM, \& Shortall AC (2008) Cure width potential for MOD resin composite molar restorations Dental Materials 24(8) 1083-1094. doi: 10.1016/j.dental.2008.01.001

25. Haenel T, Hausnerova B, Steinhaus J, Price RB, Sullivan B, \& Moeginger B (2015) Effect of the irradiance distribution from light curing units on the local microhardness of the surface of dental resins Dental Materials 31(2) 93-104. doi:10.1016/j.dental.2014.11.003

26. Brandt WC, de Moraes RR, Correr-Sobrinho L, Sinhoreti MA, \& Consani S (2008) Effect of different photoactivation methods on push out force, hardness and cross-link density of resin composite restorations Dental Materials 24(6) 846-850. doi:10.1016/j.dental.2007.09.012

27. Goncalves F, Calheiros FC, Witzel MF, Kawano Y, \& Braga RR (2007) Effect of photoactivation protocol and radiant exposure on monomer conversion and flexural strength of a resin composite after water and ethanol storage Journal of Biomedical Material Research Part B Applied Biomaterials 82(1) 89-92. doi:10.1002/jbm.b. 30708

28. Corciolani G, Vichi A, Davidson CL, \& Ferrari M (2008) The influence of tip geometry and distance on light-curing efficacy Operative Dentistry 33(3) 325-331. doi:10.2341/ 07-94

29. Zhu S \& Platt J (2011) Curing efficiency of three different curing modes at different distances for four composites Operative Dentistry 36(4) 362-371. doi:10.2341/09-245-L

30. Andre CB, Nima G, Sebold M, Giannini M, \& Price RB (2018) Stability of the light output, oral cavity tip accessibility in posterior region and emission spectrum of light-curing units Operative Dentistry 43(4) 398-407. doi:10.2341/17-033-L

31. Jandt KD \& Mills RW (2013) A brief history of LED photopolymerization Dental Materials 29(6) 605-617. doi: 10.1016/j.dental.2013.02.003

32. Musanje L \& Darvell BW (2006) Curing-light attenuation in filled-resin restorative materials Dental Materials 22(9) 804-817. doi:10.1016/j.dental.2005.11.009
33. Par M, Repusic I, Skenderovic H, Sever EK, Marovic D, \& Tarle Z (2018) Real-time light transmittance monitoring for determining polymerization completeness of conventional and bulk fill dental composites Operative Dentistry 43(1) E19-E31. doi:10.2341/17-041-L

34. Palin WM, Leprince JG, \& Hadis MA (2018) Shining a light on high volume photocurable materials Dental Materials 34(5) 695-710. doi:10.1016/j.dental.2018.02.009

35. Soh MS, Yap AU, \& Siow KS (2004) Comparative depths of cure among various curing light types and methods Operative Dentistry 29(1) 9-15.

36. Leprince JG, Leveque P, Nysten B, Gallez B, Devaux J, \& Leloup G (2012) New insight into the "depth of cure" of dimethacrylate-based dental composites Dental Materials 28(5) 512-520. doi:10.1016/j.dental.2011.12.004

37. Dewaele M, Truffier-Boutry D, Devaux J, \& Leloup G (2006) Volume contraction in photocured dental resins: the shrinkage-conversion relationship revisited Dental Materials 22(4) 359-365. doi:10.1016/j.dental.2005.03.014

38. Li J, Li H, Fok AS, \& Watts DC (2009) Multiple correlations of material parameters of light-cured dental composites Dental Materials 25(7) 829-836. doi:10.1016/j. dental.2009.03.011

39. Ferracane JL, Mitchem JC, Condon JR, \& Todd R (1997) Wear and marginal breakdown of composites with various degrees of cure Journal of Dental Research 76(8) $1508-1516$.

40. Ferracane JL (1994) Elution of leachable components from composites Journal of Oral Rehabilitation 21(4) 441-452.

41. Vandewalle KS, Ferracane JL, Hilton TJ, Erickson RL, \& Sakaguchi RL (2004) Effect of energy density on properties and marginal integrity of posterior resin composite restorations Dental Materials 20(1) 96-106.

42. Santini A, Miletic V, Swift MD, \& Bradley M (2012) Degree of conversion and microhardness of TPO-containing resin-based composites cured by polywave and monowave LED units Journal of Dentistry 40(7) 577-584. doi:10.1016/j.jdent.2012.03.007

43. Dewaele M, Asmussen E, Peutzfeldt A, Munksgaard EC, Benetti AR, Finne G, Leloup G, \& Devaux J (2009) Influence of curing protocol on selected properties of lightcuring polymers: degree of conversion, volume contraction, elastic modulus, and glass transition temperature Dental Materials 25(12) 1576-1584. doi:10.1016/j.dental. 2009.08.001

44. Moore BK, Platt JA, Borges G, Chu TM, \& Katsilieri I (2008) Depth of cure of dental resin composites: ISO 4049 depth and microhardness of types of materials and shades Operative Dentistry 33(4) 408-412. doi:10.2341/07-104

45. Vasudeva G (2009) Monomer systems for dental composites and their future: a review $J$ California Dental Association 37(6) 389-398.

46. Miletic V \& Santini A (2012) Micro-Raman spectroscopic analysis of the degree of conversion of composite resins containing different initiators cured by polywave or monowave LED units Journal of Dentistry 40(2) 106-113. doi:10.1016/j.jdent.2011.10.018 
47. Ilie $\mathrm{N}$ (2017) Impact of light transmittance mode on polymerisation kinetics in bulk-fill resin-based composites Journal of Dentistry 63 51-59. doi:10.1016/j.jdent. 2017.05.017

48. Sim JS, Seol HJ, Park JK, Garcia-Godoy F, Kim HI, \& Kwon YH (2012) Interaction of LED light with coinitiatorcontaining composite resins: effect of dual peaks Journal of Dentistry 40(10) 836-842. doi:10.1016/j.jdent.2012.06. 008
49. Lucey SM, Santini A, \& Roebuck EM (2015) Degree of conversion of resin-based materials cured with dual-peak or single-peak LED light-curing units International Journal of Paediatric Dentistry 25(2) 93-102. doi:10. 1111/ipd.12104

50. Mousavinasab SM \& Meyers I (2011) Comparison of depth of cure, hardness and heat generation of LED and high intensity QTH light sources European Journal of Dentistry 5(3) 299-304. 